УДК 519.876.2:336

DOI: $10.25140 / 2411-5363-2019-2(16)-86-94$

\title{
Тетяна Нескородева
}

\section{МЕТОДИКА ФОРМАЛИЗАЦИИ ЗАДАЧ СИСТЕМЫ АУДИТА ПЕРВОГО УРОВНЯ}

\begin{abstract}
Актуальность темы исследования. В настоямее время актуальной научно-технической проблемой информационных технологий финансово-экономической сферы является автоматизачия анализа данных финансовоэкономической информации предприятий. Решение задач аудита на основании автоматизированного анализа данных является одной из актуальных проблем. Поэтому существует потребность создания методики проектирования информационных технологий (ИТ) систем поддержки принятия решений (СППР) аудита.

Постановка проблемы. При проектировании ИТ аудита необходимо обеспечить возможность их применения к финансово-экономическим данным предприятий различных отраслей, типов производства, различной организационной структурой и ИТ учета.

Анализ последних исследований и публикаций. Были рассмотрены последние публикации в открытом доступе, включая технологию системы Aиdit 4.0.

Выделение неисследованных частей общей проблемы. Автоматизация решения задач аудита предпосылки бухгалтерского учета инвариантна относительно особенностей предприятия.

Постановка задачи. Создание методики формализачии задач аудита предпосылки бухгалтерского учета инвариантной относительно особенностей предприятия.

Изложение основного материала. Предлагаемая методика формализачии задач основана на правилах построения взаимно-однозначного отображения данных подчиненного проверяемой предпосылке и формализации взаимосвязей переменных первичного учета, характеризующих правила предпосылки.

Выводы по статье. Предложенная методика формализачии задач аудита позволяет использовать их для автоматизации решения задач аудита широкого класса предприятий.

Ключевые слова: формализачия задач аудита; информачионная технология; СППР аудита; взаимнооднозначное отображение; первичный учет.

Табл.: 1. Библ.: 12
\end{abstract}

Актуальность темы исследования. В настоящее время актуальной научнотехнической проблемой информационных технологий финансово-экономической сферы является автоматизация анализа больших объемов данных финансовоэкономической информации предприятий, хранящихся и поступающих в режиме онлайн в базы данных локальных и глобальных компьютерных систем с целью формирования рекомендаций принятия решений при аудите. Так, например, эффективность ИТ бизнеса в сокращении коррупции обоснована в [1]. В [2] рассмотрены теоретические и практические вопросы, связанные с применением ИТ в аудите. Определены изменения, которые вносит в методологию аудита его комплексная компьютеризация.

Применение современных систем учета и управления, на предприятии которые интегрированы в глобальные, многоуровневые системы информационных компьютерных сетей дает потенциальные возможности обработки больших объемов данных, которые используются не в полной мере [3]. В [4] представлена архитектура подсистем внешнего и внутреннего аудита системы Audit 4.0, внедрение и функционирование которых сталкивается с проблемой обработки больших объемов данных. Также на сегодняшний день рассматривается возможность принятия законодательных актов, которые позволят использовать аналитические доказательства (в частности противоречивости данных) полученные при анализе больших объемов данных в качестве обоснования судебных решений без документального исследования первичных документов [5]. Это свидетельствует, что тематика исследований, посвященных проектированию ИТ аудита является актуальной.

Постановка проблемы. При проектировании ИТ аудита необходимо предполагать возможность их применения к финансово-экономическим данным предприятий различных отраслей, типов производства, различной организационной структурой и ИТ учета. Экономико-производственная деятельность и ИТ предприятия образуют сложную социоэкономическую техническую систему. Данная система, как объект аудита, характеризуется глобальной многоуровневой иерархичной структурой разнородных, многофакторных, многофункциональных связей, взаимозависимостей и взаимодействий его подси-

(C) Нескородєва Т. В., 2019 
TECHNICAL SCIENCES AND TECHNOLOGIES

стем, с ИТ контроля, учета, управления, ведения бизнеса, с другими ИТ и системами в структуре информационной системы национальной экономики. При этом на каждом уровне данные имеют сетевую структуру. Современная методология прикладного системного анализа не соответствует данным требованиям [6]. Следовательно, существует проблема разработки методологических основ проектирования ИТ аудита.

Анализ последних исследований и публикаций. Говоря об автоматизации процедур анализа, следует отметить, что в литературе представлены методы обнаружения мошенничества, основанные на применении моделей нейронных (вероятностных) сетей, логистических регрессий, деревьях решений, экспертных оценок, статистических методах анализа данных [7-10]. Методы анализа, предложенные в [2, 10], позволяют автоматизировать в ИТ аудита только отдельные процедуры анализа (например, формирование выборки) или провести аналитические процедуры в специальных случаях. То есть существует проблема полноты, универсальности и комплексности методик ИТ анализа.

В информационных системах бухучета учет данных одного вида объекта и одного вида операции осуществляется в автоматическом режиме. При этом взаимосвязи между объектами и операциями разных видов учитываются в ручном режиме. Для автоматизации проверки данных взаимосвязей была предложена методика обобщенномножественного отображения информации в [11].

Выделение неисследованных частей общей проблемы. Проектирование ИТ СППР согласно данной методики требует создания методики формализации информации первичного учета для автоматизированного решения задач аудита предпосылок П(с)БУ. В [12] выполнена постановка задач автоматизированного анализа данных в подсистеме аудита предпосылки в виде проверки отображения соответствующих множеств данных элементарных задач за период проверки. Это позволяет перейти к формализации задач подсистем ИТ СППР аудита.

Цель статьи. Создать методику формирования взаимно-однозначного отображения множеств данных задач аудита предпосылки за период проверки инвариантную относительно особенностей предприятия и как составляющих методики формализации информации при проектировании ИТ.

Для этого необходимо решить следующие задачи:

1) построение взаимно-однозначного отображения номеров подсистем первичного учета (ППУ) на основании отношений соответствия в БД ИТ учета;

2) преобразование выходных переменных задач аудита в соответствии с взаимнооднозначным отображением номеров ППУ;

3) определение совокупностей взаимосвязей переменных;

4) выделение взаимосвязей переменных ППУ, характеризующих правила предпосылки;

5) формирование взаимно однозначного отображения данных, подчиненного правилам предпосылки.

Методики в предыдущих работах автора [11; 12] проиллюстрированы на примере задач подсистемы аудита предпосылки «Полнота» материальных расходов. Поэтому в данной работе методика будет рассмотрена на примере первой задачи аудита расходов проверки отображения «оплачено-получено».

Изложение основного материала.

1. Построение взаимно-однозначного отображения номеров ППУ.

В задаче проверки отображения «оплачено-получено» платежные поручения (ПП) образуют множество ППУ входных данных подсистемы «Расчеты с поставщиками». Товарно-транспортные накладные (ТТН) образуют множество ППУ выходных данных. Согласно этому определим входные и выходные переменные подсистемы «Расчеты с поставщиками». 
Вектор входных переменных подсистемы ИТ включает показатель номера ПП $n$ и показатели ПП:

$$
\bar{X}=\left(\left(n, \delta_{d}, p_{d}, \Theta_{d},\left(v_{S_{d}}, c_{S_{d}}, \delta_{S_{d}}\right), s_{d} \in \Theta_{d}, d a t a_{d}\right), n \in N\right),
$$

где переменные определены в табл. 1 (1 столбец).

Вектор выходных переменных подсистемы ИТ включает показатели номера ТTH $m$ и номера $l$ ПП, которое находится в отношении соответствия с данной ТТН. Следовательно, номер ПП $l$ является образом при отображении $\varepsilon$ номера ТТН, которое для данной подсистемы назовем «получено-оплачено»:

$$
l=\varepsilon(m),
$$

где $\varepsilon$ - отображение множества номеров ТТН на множество номеров ПП.

Переменные учета ТТН табл. 1 (2 столбец):

$$
\bar{Y}=\left(\left(m, l, \Theta_{k}, \delta_{k}, p_{k},\left(v_{S_{k}}, c_{S_{k}}, \delta_{S_{k}}\right), s_{k} \in \Theta_{k}, \text { data }_{k}\right), m \in \mathrm{M}\right),
$$

где $l$ - номер ПП, которое находится в отношении соответствия «получено-оплачено» с ТТН с номером $m$, остальные переменные определены в табл. 1 (2 столбец).

\begin{tabular}{|c|c|c|c|}
\hline \multicolumn{2}{|c|}{ Входные переменные» } & \multicolumn{2}{|c|}{ Выходные переменные } \\
\hline обозначение $x_{63}^{i}$ & название & обозначение $y_{63}^{j}$ & название \\
\hline$d$ & $\begin{array}{c}\text { вид операции (оплата счета } \\
\text { поставщика) }\end{array}$ & $k$ & $\begin{array}{c}\text { вид операции (получение сырья } \\
\text { от поставщика) }\end{array}$ \\
\hline$p_{d}$ & $\begin{array}{c}\text { вид поставщика, которому } \\
\text { переведена оплата }\end{array}$ & $p_{k}$ & $\begin{array}{c}\text { вид поставщика, который поста- } \\
\text { вил сырье }\end{array}$ \\
\hline$S_{d}$ & вид оплаченного сырья & $S_{k}$ & вид полученного сырья \\
\hline$\theta_{d}$ & $\begin{array}{c}\text { множество видов оплаченного } \\
\text { сырья }\end{array}$ & $\theta_{k}$ & $\begin{array}{c}\text { множество видов полученного } \\
\text { сырья }\end{array}$ \\
\hline$q_{d}$ & $\begin{array}{c}\text { количество видов оплаченного } \\
\text { сырья }\end{array}$ & $q_{k}$ & $\begin{array}{c}\text { количество видов полученного } \\
\text { сырья }\end{array}$ \\
\hline$v_{s_{d}}$ & $\begin{array}{l}\text { количество оплаченного сырья } \\
\qquad \text { вида } S_{d}\left(S_{d} \in \Theta_{d}\right)\end{array}$ & $v_{s_{k}}$ & $\begin{array}{l}\text { количество полученного сырья } \\
\text { вида } S_{k}\left(S_{k} \in \Theta_{k}\right)\end{array}$ \\
\hline$c_{s_{d}}$ & $\begin{array}{l}\text { цена оплаченного сырья вида } \\
\qquad S_{d}\left(S_{d} \in \Theta_{d}\right)\end{array}$ & $C_{s_{k}}$ & $\begin{array}{l}\text { цена полученного сырья вида } S_{k} \\
\qquad\left(S_{k} \in \Theta_{k}\right)\end{array}$ \\
\hline$\delta_{s_{d}}$ & $\begin{array}{l}\text { стоимость оплаченного сырья } \\
\text { вида } S_{d}\left(S_{d} \in \Theta_{d}\right)\end{array}$ & $\delta_{s_{k}}$ & $\begin{array}{l}\text { стоимость полученного сырья } \\
\text { вида } S_{k}\left(S_{k} \in \Theta_{k}\right)\end{array}$ \\
\hline$\delta_{d}$ & $\begin{array}{c}\text { стоимость оплаченного сырья } \\
\text { множества } \Theta_{d}\end{array}$ & $\delta_{k}$ & $\begin{array}{c}\text { стоимость полученного сырья } \\
\text { множества } \Theta_{k}\end{array}$ \\
\hline
\end{tabular}

Таблица 1

Переменные учета расчетов с поставщиками

В силу экономического содержания взаимодействия операций оплаты и поставки каждому номеру ТТН $m(m \in M)$ соответствует единственный номер $l(l \in L)$ ПП, то данное соответствие номеров за период $T$ порождает отображение множеств номеров:

$$
\mathrm{M} \stackrel{\varepsilon}{\longrightarrow} L,
$$

где $\varepsilon$ - отображение номеров выходных данных на номера входных данных;

$\mathrm{L}$ - множество номеров ПП в ТTH. 
TECHNICAL SCIENCES AND TECHNOLOGIES

В каждой ТТН с номером $m$ учитывается соответствие единственной ПП с номером l. Следовательно, существует отображение номеров ТТН на входные (например, «получено-оплачено» в подсистеме «Расчеты с поставщиками»):

$$
\forall m \in \mathrm{M} \exists ! l: m \stackrel{\varepsilon}{\longrightarrow} l,
$$

где $l$ - номер первичной подсистем учета входных данных в ПП;

$m$ и $M-$ номер и множество номеров ТТН;

$\varepsilon$ - отображение множества номеров ТТН на номера ПП, между которыми существует соответствие к одному множеству объектов учета.

Отображение $\varepsilon$ является однозначным, но не является взаимно-однозначным. Для проверки предпосылки «Полнота» при преобразовании входных данных в выходные за период $T$, которое назовем «оплачено-получено» на основании образа отображения $\varepsilon$ номеров первичных подсистем «получено-оплачено» в БД ИТ учета сформируем взаимно однозначные отображение.

На первом этапе формирования отображения определим множества ПП, для которых нет ТTН, которые находятся в отношении соответствия к одному объекту учета и наоборот за период $T$. Из (1) следует, образ множества номеров выходных данных $\varepsilon(\mathrm{M})=L$ определяет множество номеров ПП которые в системе учета определены как прообраз выходных данных за период $T$. Возможны три случая при сопоставлении данного множества с множеством номеров $N$ учтенных ПП.

Первый - существуют ПП для ТТН в период $T$, которые находятся в отношении соответствия к одному объекту:

$$
(l \in L) \wedge(l \in N) .
$$

Второй - не существует ПП для ТТН в период $T$, которые находятся в отношении соответствия к одному объекту:

$$
(l \in L) \wedge(l \notin N)
$$

Совокупность номеров ТТН, образ которых при отображении $\varepsilon$ удовлетворяет условию (3) образуют неэквивалентное множество при отображении $\varepsilon$ (по фактам поставкаоплата за период $T$ ), которое обозначим:

$$
M_{H}=\left\{m_{l} \in M_{l}: l \notin N\right\} .
$$

Третий - не существует ТТН для ПП в период $T$, которые находятся в отношении соответствия к одному объекту:

$$
(l \in N) \wedge(l \notin L) .
$$

Тогда разность множеств номеров $N$ ПП, учтенных за период $T$ и номеров ППУ, которые являются образом $L$ при отображении $\varepsilon$ определяет множество номеров ПП неэквивалентных на при данном отображении (оплата-поставка за период $T$ ).

$$
N_{H}=N \backslash L .
$$

На втором этапе множество номеров $M \backslash M_{н}$ ТТН, для которых существуют образы при отображении $\varepsilon$, представим в виде системы непересекающихся подмножеств, образы которых различны:

$$
\mu=\left\{M_{l}, l \in L \cap N\right\}
$$

Так как отображение $\varepsilon$ является однозначным, то прообразы различных ПП в (9) не пересекаются: 


$$
M_{l_{1}} \cap M_{l_{2}}=\varnothing, l_{1} \neq l_{2} .
$$

Определим взаимно-однозначное отображение «оплачено-получено» номеров ПП на разбиение множеств номера ППУ выходных в БД:

$$
\phi: N \cap L \rightarrow \mu .
$$

2. Преобразование выходных переменных в соответствии с взаимно однозначным отображением номеров ППУ. Для определения образа входных данных $X$ при отображении $\phi$ номеров первичных подсистем (11), выполним преобразование выходных переменных вектора (2) согласно отношения соответствия:

$$
\begin{aligned}
& P_{k}\left(M_{l}\right)=\bigcup_{m_{l} \in M_{l}} p_{k}\left(m_{l}\right), \theta_{k}\left(M_{l}\right)=\bigcup_{m_{l} \in M_{l}} \theta_{k}\left(m_{l}\right), C_{s_{k}}\left(M_{l}\right)=\bigcup_{m_{l} \in M_{l}} c_{s_{k}}\left(m_{l}\right), \\
& v_{s_{k}}\left(M_{l}\right)=\sum_{m_{l} \in M_{l}} v_{s_{k}}\left(m_{l}\right), \delta_{s_{k}}\left(M_{l}\right)=\sum_{m_{l} \in M_{l}} \delta_{s_{k}}\left(m_{l}\right), \delta_{k}\left(M_{l}\right)=\sum_{m_{l} \in M_{l}} \delta_{k}\left(m_{l}\right) .
\end{aligned}
$$

Тогда преобразованный, вектор выходных переменных примет вид:

$$
\overline{Y^{\varepsilon}}=\left\{\left(M_{l}, P_{k}\left(M_{l}\right),\left(\theta_{k}\left(M_{l}\right), \delta_{k}\left(M_{l}\right)\right),\left(\delta_{s_{k}}\left(M_{l}\right), C_{s_{k}}\left(M_{l}\right)\right), s_{k} \in \Theta_{k}, \bigcup_{l \in N} M_{l}=\mathrm{M}\right\},\right.
$$

где переменные определены выше.

3. Выделение взаимосвязей переменных ППУ, характеризующих правила предпосылки и определение функциональных особенностей их учета. Между переменными подсистемы (1), (2) и (12) существует множество взаимосвязей. Выделим подмножество взаимосвязей между:

1) входными переменными (1) - $\Phi_{X}$,

2) выходными переменными (2) - $\Phi_{Y}$;

3) выходными преобразованными переменными (12) - $\Phi_{Y^{\varepsilon}}$;

4) входными и выходными переменными (1) и (2) до установления отношения соответствия «оплачено-получено» между номерами ППУ - $\Phi_{X}^{Y}$;

5) входными и выходными переменными (1) и (12) после определения отображения «оплачено-получено» между номерами ППУ $-\Phi_{X}^{Y^{\varepsilon}}$.

Проверка предпосылки формализована в виде установления взаимно однозначности отображения между входными и выходными данными. Следовательно, взаимосвязи между входными и выходными переменными $\Phi_{X}^{Y}$ и $\Phi_{X}^{Y^{\varepsilon}}$ характеризуют правила предпосылки.

Функциональные особенности учета данных множеств взаимосвязей на первом уровне заключаются в ручном режиме их осуществления.

4. Формирование отображения подчиненного правилам предпосылки. Реализация взаимосвязей $\Phi_{X}^{Y^{\varepsilon}}$ входных переменных подсистемы учета (1) и выходных (12) после преобразования согласно отображению $\phi$ номеров ПП на ТТН (например, «оплачено-получено») порождает взаимно-однозначное отображение множеств их значений:

$$
X^{\varepsilon} \stackrel{\gamma}{\longrightarrow} Y^{\varepsilon},
$$

где где $X^{\varepsilon}$ - значения входных переменных $\bar{X}$ в ППУ множества $N \cap L, Y^{\varepsilon}-$ значения преобразованных в соответствии с отображением $\varepsilon$ номеров ППУ за период $T$ вы- 
TECHNICAL SCIENCES AND TECHNOLOGIES

ходных переменных вектора $\overline{Y^{\varepsilon}} ; \gamma$ - отображение входных данных ППУ на выходные (например, «оплачено-получено») после определения отображения $\varepsilon$ номеров ППУ.

Согласно, постановке задачи проверки предпосылки «Полнота» [...], пары множеств $(X, Y)$ и $\left(X^{\varepsilon}, Y^{\varepsilon}\right)$ подлежат анализу с целью выделения эквивалентных и неэквивалентных подмножеств при отображении «оплачено-получено» $\gamma$. Поэтому правила отображения формализуем в виде взаимосвязей переменных, которые характеризуют предпосылку.

5. Методика формализации взаимосвязей переменных первичного учета, характеризующих правила предпосылки. Согласно правилу предпосылки «Полнота» все, что оплачено за период $T$ должно быть получено за период $T$ и наоборот. Определим взаимосвязи, характеризующие правила предпосылки «Полнота» при учете расчетов с поставщиками. Для этого выделим две совокупности условий:

1) $K_{2}^{(1)}$ для множеств номеров ПП и ТТН в подсистеме «Расчеты с поставщиками» $N$ и $M$;

2) $K_{2}^{(2)}$ для множеств значений переменных ПП и ТТН в подсистеме «Расчеты с поставщиками», для которых выполняется условие $K_{2}^{(1)}$.

Множества $N$ и $M$ должны удовлетворять трем условиям, характеризующим правила предпосылки «Полнота»:

$$
\begin{aligned}
& \forall l \in N \exists M_{l} \in \mu:\left(M_{l} \neq \varnothing\right) \wedge\left(\forall m \in M_{l} \Rightarrow \varepsilon(m)=l\right), \\
& \bigcup_{l \in N} M_{l}=\mathrm{M}, M_{l_{1}} \cap M_{l_{2}}=\varnothing, l_{1} \neq l_{2} .
\end{aligned}
$$

Обозначим систему множеств номеров порожденных отображением $\varepsilon$ :

$$
\mu=\left\{M_{l}, l \in N \cap L\right\} .
$$

Т.е. должно существовать взаимно-однозначное отображение множеств номеров ПП на систему множеств номеров ТТН (20), порожденных отображением $\varepsilon$ :

$$
\phi: N \cap L \rightarrow \mu \text {. }
$$

Условия (18), (19) образуют первую совокупность условий предпосылки «Полнота» $K_{2}^{(1)}$. Для ППУ, которые удовлетворяют условию $K_{2}^{(1)}$ определим вторую совокупность условий предпосылки «Полнота» $K_{2}^{(2)}$. Условия предпосылки формализуем в виде взаимосвязей переменных операций, которые находятся в отношении соответствия к одному объекту учета $e_{s}$ «Расчеты с поставщиками»:

$$
\begin{aligned}
& \delta_{d}(l)=\delta_{k}\left(M_{l}\right), \Theta_{d}(l)=\Theta_{k}\left(M_{l}\right), \forall l: l \in N, \\
& p_{d}(l)=p_{k}\left(m_{l}\right), m_{l} \in M_{l}, \forall l: l \in N, \\
& v_{s_{d}}(l)=v_{s_{k}}\left(M_{l}\right), \delta_{s_{d}}(l)=\delta_{s_{k}}\left(M_{l}\right), s_{d} \in \Theta_{d}, s_{d}=s_{k}, \forall l: l \in N, \\
& c_{s_{d}}(l)=c_{s_{k}}\left(m_{l}\right), m_{l} \in M_{l}, s_{d} \in \Theta_{d}, s_{d}=s_{k}, \forall l: l \in N .
\end{aligned}
$$

Взаимосвязи (16) - (19) и (20) - (23) переменных, которые порождают отображение «оплачено-получено» являются формализацией правил предпосылки «Полнота» $K_{2}^{(1)}$ и $K_{2}^{(2)}$ в подсистеме «Расчеты с поставщиками». 
6. Формализация задач подсистем аудита предпосылки в ИТ СППР аудита. На основании выполненной формализации правил предпосылки выполним формализацию задач подсистемы аудита предпосылки.

Для этого задачи обработки данных в подсистемах аудита предпосылок на различных этапах работы системы разделим на задачи двух видов по целевому назначению.

1. Задача первого целевого назначения - определить первичные подсистемы, для данных которых условия $K_{2}^{(1)}$ или $K_{2}^{(2)}$ не выполняются.

Определить подмножества номеров первичных подсистем учета входных и выходных данных $N_{H}$ и $M_{H}$ и соответственно за период проверки $T$ таких, что $\left(H_{d}, H_{k}\right)$ - данные выделенных подсистем не удовлетворяют условиям эквивалентности $K_{2}^{(1)}$ или $K_{2}^{(2)}$ для предпосылки «Полнота» $\Pi_{2}$ :

$$
\begin{gathered}
\exists\left(N_{H} \subseteq N\right) \wedge\left(M_{H} \subseteq \mathrm{M}\right):\left(K_{2}^{(1)}\left(H_{d}, H_{k}\right)=0\right) \vee\left(K_{2}^{(2)}\left(H_{d}, H_{k}\right)=0\right), \\
H_{d}=X\left(N_{H}\right), H_{k}=Y\left(M_{H}\right),
\end{gathered}
$$

где $\left(N_{H}, M_{H}\right)$ - пара подмножеств номеров ППУ неэквивалентных входных и выходных данных при отображении по правилам предпосылки «Полнота» $\Pi_{2}$;

$\left(H_{d}, H_{k}\right)$ - пара неэквивалентных подмножеств входных и выходных данных предпосылки «Полнота» $\Pi_{2}$.

2. Задача второго целевого назначения - определить подмножества первичных подсистем, для которых условия предпосылки $K_{2}^{(1)}$ и $K_{2}^{(2)}$ выполняются.

Определить подмножества номеров ППУ входных и выходных данных за период проверки $T N_{e}$ и $M_{e}$ таких, что множества $\mathrm{E}_{d}$ и $\mathrm{E}_{k}$ данных подсистем соответственно удовлетворяют совокупности условий $K_{2}^{(1)}$ и $K_{2}^{(2)}$ предпосылки «Полнота» $\Pi_{2}$ :

$$
\begin{gathered}
\exists\left(N_{e} \subseteq N\right) \wedge\left(M_{e} \subseteq \mathrm{M}\right): K_{2}\left(\mathrm{E}_{d}, \mathrm{E}_{k}\right)=1, \\
K_{2}=K_{2}^{(1)} \wedge K_{2}^{(2)}, \mathrm{E}_{d}=X\left(N_{e}\right), \mathrm{E}_{k}=Y\left(M_{e}\right),
\end{gathered}
$$

где $\left(N_{e}, M_{e}\right)$ - пара подмножеств номеров ППУ эквивалентных входных и выходных данных предпосылки «Полнота»;

$\left(\mathrm{E}_{d}, \mathrm{E}_{k}\right)$ - пара эквивалентных подмножеств входных и выходных данных предпосылки «Полнота».

Методика предполагает, что определение подмножеств ППУ входных и выходных данных за период проверки $T$, данные которых подлежат сплошной проверке с целью определения их эквивалентности осуществляется на основании предварительного этапа обобщенно-множественного анализа.

Выводы по статье. Выполненная формализация задач подсистем аудита предпосылки в ИТ СППР аудита инвариантна относительно особенностей функционирования и системы учета предприятия, что позволяет использовать их для автоматизации решения задач аудита.

\section{Список использованных источников}

1. Xinli Hu. Effectiveness of information technology in reducing corruption in China. Electronic Library. 2015. Vol. 33. Issue 1. P. 52-64. DOI: 10,1108/el-11-2012-0148.

2. Ивахненков С. В. Информационные технологии аудита и внутрихозяйственного контроля в контексте мировой интеграции: монография. Житомир: ЧП «Рута», 2010. 432 с. 
TECHNICAL SCIENCES AND TECHNOLOGIES

3. Kirkos E., Spathis C., Manolopoulos Y. Data mining techniques for the detection of fraudulent financial statement. Expert Syst. Appl. 2007. Vol. 32. Issue 4. P. 995-1003.9. DOI: 10.1016/j.eswa.2006.02.016.

4. Dai J, Vasarhelyi M. A. Imagineering Audit 4.0. Journal of Emerging Technologies in Accounting. 2016. № 13(1). P. 1-15.

5. Яремко С. А., Коваленко В. В. Дослідження проблем впровадження сучасних інформаційних систем аудиту в контрольно-ревізійній діяльності. Комп'ютерно-інтегровані технологіï: освіта наука виробництво. Луцьк, 2014. № 14. С. 179-182.

6. Згуровський М. 3., Панкратова Н. Д. Системний аналіз: Методологія. Проблеми. Додатки: монографія. 2-ге видання, перероблене та доповнене. Київ: Наукова думка, 2016. 726 с.

7. Jarrod West, M. Bhattacharya and R Islam. Intelligent Financial Fraud Detection Practices: a comprehensive review. Computers \& Security. 2016. Vol. 57. P. 47-66. DOI: 10.1007/978-3-31923802-9 16.

8. Chi-Chen Lin, Chiu An-An, Yan Huang Shaio, C. Yen. David. Detecting the financial statement fraud: The analysis of the differences between data mining techniques and experts' judgments. Knowledge-Based Systems. 2015. № 89- P. 459-470. DOI: 10.1016/j.knosys.2015.08.011.

9. Mohiuddin Ahmeda, N. Mahmooda Abdun, Md Rafiqul Islam. A survey of anomaly detection techniques in financial domain. Future Generation Computer Systems. 2016. № 55. P. 278-288.

10. Бюль А., Цёфель П. SPSS: Искусство обработки информации. Анализ статистических данных и восстановление скрытых закономерностей: пер. с нем. Санкт-Петербург: ДиаСофтЮП, 2005. 608 с.

11. Нескородева, Т. В. Правила и составные части методики обобщенно-множественного отображения информации в подсистеме аналитического учета СППР аудита верхнего уровня. Вісник НТУ «ХПІ». Серія: Системний аналіз, управління та інформаційні технологї̈. 2017. № 55 (1276). C. 31-38.

12 Нескородєва Т. В. Постановка елементарних задач аудиту передумови положень бухгалтерського обліку в інформаційній технології системи підтримки прийняття рішень. Сучасні інформаиійні системи. 2019. Т. 3, № 1. C. 48-53. DOI: 10.20998/2522-9052.2019.1.08.

\section{References}

1. Xinli, Hu. (2015). Effectiveness of information technology in reducing corruption in Chin. Electronic Library, 33 (1), 52-64. DOI: 10,1108/el-11-2012-0148.

2. Ivakhnenkov, S. V. (2010). Informatsionnye tekhnologii audita i vnutrikhoziaistvennogo kontrolia $\mathrm{v}$ kontekste mirovoi integratsii [Information technology of the audit and internal control in the context of world integration]. Zhitomir: $\mathrm{ChP}$ «Ruta» Publ. [in Russian].

3. Kirkos, E., Spathis, C., Manolopoulos, Y. (2007). Data mining techniques for the detection of fraudulent financial statement. Expert Syst. Appl, 32 (4), 995-1003.9. DOI: 10.1016/j.eswa.2006.02.016.

4. Dai, J, Vasarhelyi, M. A. (2016). Imagineering Audit 4.0. Journal of Emerging Technologies in Accounting, 13(1), 1-15 [in English].

5. Yaremko, S. A., Kovalenko, V. V. (2014). Doslidzhennia problem vprovadzhennia suchasnykh informatsiinykh system audytu $\mathrm{v}$ kontrolno-reviziinii diialnosti [Investigation of the problems of implementation of modern information audit systems in the control and audit activity]. Kompiuternointehrovani tekhnolohii: osvita nauka vyrobnyctvo - Computer Integrated Technologies: Education, Science, Production, 14, 179-182 [in Ukrainian].

6. Zhurovskyi, M. Z., Pankratova, N. D. (2016). Systemnyi analiz: Metodolohiia. Problemy. Prylozhennia [System Analysis: Methodology. Problems Applications. Monograph 2nd edition, revised and supplemented] ( $2^{\text {nd }}$ ed.). Kyiv: Naukova dumka [in Ukrainian].

7. Jarrod, West, Bhattacharya, M. and Islam, R. (2016). Intelligent Financial Fraud Detection Practices: a comprehensive review. Computers \& Security, 57, 47-66. DOI: 10.1007/978-3-31923802-9 16.

8. Chi-Chen, Lin, Chiu, An-An, Shaio, Yan Huang and David, C. Yen. (2015). Detecting the financial statement fraud: The analysis of the differences between data mining techniques and experts' judgments. Knowledge-Based Systems, 89, 459-470 [in English]. 
9. Mohiuddin Ahmeda, Mahmooda Abdun N and Rafiqul Islam Md. (2016). A survey of anomaly detection techniques in financial domain. Future Generation Computer Systems, 55, 278-288. DOI: 10.1016/j.knosys.2015.08.011.

10. Biul, A., Tsefel, P. (2005). SPSS: Iskusstvo obrabotki informatsii. Analiz statisticheskikh dannykh $i$ vosstanovlenie skrytykh zakonomernostei [SPSS: The art of information processing. Analysis of statistical data and restoration of hidden patterns]. St. Petersburg: DiaSoftJuP [in Russian].

11. Neskorodeva, T. V. (2017). Pravila i sostavnye chasti metodiki obobshhennomnozhestvennogo otobrazheniia informatsii v podsisteme analitcheskogo ucheta SPPR audita verkhnego urovnia [Rules and components of a generalized-multiple display method of information in the express analysis subsystem of the audit decision support system]. Visnyk NTU «KhPI». Seriia: Sistemnyi analiz, upravlinnia ta informatsiini tekhnologii - Bulletin of NTU "KhPI". Seriya: System Analysis, Management and Informacion Technology, 55 (1276), 31-38 [in Russian].

12. Neskorodeva, T. B. (2019). Postanovka elementarnykh zadach audytu peredumovy polozhen bukhhalterskoho obliku $\mathrm{v}$ informatsiinii tekhnolohii systemy pidtrymky pryiniattia rishen [Formulation of elementary tasks of audit subsystems of accounting provisions precondition IT DSS]. Suchasni informatsiini systemy - Modern Information Systems, 3 (1), 48-53. DOI: 10.20998/2522-9052.2019.1.08.

UDC 519.876.2:336

\section{Tatiana Neskorodieva}

\section{TASK FORMALIZATION METHOD OF THE FIRST LEVEL AUDIT SYSTEM}

Urgency of the research. At present, the actual scientific and technical problem of information technology in the financial and economic sphere is the automation of data analysis of financial and economic information of enterprises. The solution of audit tasks based on automated data analysis is one of the actual problems. Therefore, there is a need to create a methodology for designing DSS auditing.

Target setting. When designing an IT audit, it is necessary to ensure that they can be applied to the financial and economic data of enterprises in various industries, types of production, various organizational structures and IT accounting.

Actual scientific researches and issues analysis. The latest open source publications were reviewed, including Audit 4.0 technology.

Uninvestigated parts of general matters defining. The automatization of the tasks of the audit of the prerequisite of accounting is invariant with respect to the features of the enterprise.

The research objective. The automatization tasks of the audit of the prerequisite of accounting is invariant with respect to the features of the enterprise.

The statement of basic materials. The proposed methodology for formalizing tasks is based on the rules for constructing a one-to-one mapping of data from a subordinate to the checked premise and formalizing the interrelationships of primary accounting variables that characterize the rules of the premise.

Conclusions. The proposed methodology for formalizing audit tasks allows them to be used to automate the solution of audit tasks for a wide class of enterprises.

Keywords: automated data analysis; formalization of audit tasks; information technology; DSS audit; one-to-one mapping; primary accounting.

Table: 1. References: 12.

Нескородева Татьяна Васильевна - кандидат технических наук, доцент, доцент кафедры математики и математических методов в экономике, Донецкий национальный университет имени Василя Стуса (ул. 600-летия, 21, г. Винница 21027, Украина).

Neskorodieva Tatiana - PhD in Technical Sciences, Associate Professor, Associate Professor of the Department of mathematics and mathematical methods in Economics, Donetsk national University named Vasyl Stus (21 600-richya Str., 21027 Vinnytsia, Ukraine).

E-mail: t.neskorodieva@donnu.edu.ua

ORCID: http://orcid.org/0000-0003-2474-7697 